\title{
THE NEAT EMBEDDING PROBLEM AND THE NUMBER OF VARIABLES REQUIRED IN PROOFS
}

\author{
ROGER D. MADDUX
}

(Communicated by Andreas R. Blass)

\begin{abstract}
By constructing special relation algebras we show that if $3<\alpha<$ $\omega$, then

$$
\mathrm{SNr}_{3} \mathrm{CA}_{\alpha} \neq \mathrm{SNr}_{3} \mathrm{CA}_{3 \alpha-7}
$$

and there is a logically valid first-order sentence containing at most three variables with a proof in which every sentence has at most $3 \alpha-7$ variables, but no proof in which every sentence has at most $\alpha$ variables.
\end{abstract}

The neat embedding problem is Problem 2.12 of [HMT71], which reads: For $2<\gamma<\omega$, is there a $\kappa<\omega$ such that $\mathbf{S N r}_{\gamma} \mathrm{CA}_{\gamma+\kappa}=\mathbf{S N r}_{\gamma} \mathrm{CA}_{\gamma+\kappa+1}$ ? It is reasonable to conjecture that the answer is "no." (See $\left[\mathrm{Mo69}\right.$, p. $\left.342^{28}\right]$.) The key case is $\gamma=3$, for if the answer could be shown to be "no" for this case, then the same would be true for all other values of $\gamma$.

For every finite $\alpha>3$, let $\phi(\alpha)$ be the least $\beta$ such that $\mathrm{SNr}_{3} \mathrm{CA}_{\alpha} \neq$ $\mathrm{SNr}_{3} \mathrm{CA}_{\beta}$. Then $\phi(\alpha)$ always exists, by [Mo69]. It is known that $\phi(4)=5$ and $\phi(5)=6$. The results in [Mo69] show that $\phi(\alpha) \leq 3 \alpha$ !. This can also be shown by the method of [Ma89], which uses the same combinatorial principle as [Mo69]. If the answer to Problem 2.12 is "no," then $\phi(\alpha)=\alpha+1$. Theorem 1 below shows that $\phi(\alpha) \leq 3 \alpha-7$. The proof is based on the fact that, as the cardinality of a set $X$ increases, the number of 3-element subsets of $X$ grows faster than any constant times the number of 2-element subsets of $X$.

Let $\mathscr{M}$ be a first-order language with at least countably many relation symbols of rank at least two. It is reasonable to expect that if $3 \leq \alpha<\omega$, then there is a logically valid 3-sentence $X$ (only three variables are used in $X$, although each may appear many times) which is $\alpha$-provable (has a proof in which every sentence is an $\alpha$-sentence) but not $(\alpha+1)$-provable. If this could be proved it would also settle Problem 2.12. Using the methods and results of [Mo69], [Mo71], and [J69] (or [Ma89]), it is possible to prove that there is a 3-sentence

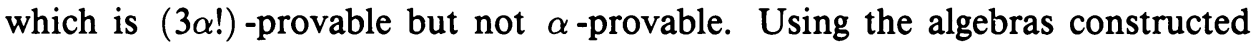
in the proof of Theorem 1, we can show if $3<\alpha<\omega$, then there is a 3sentence $X$ which is $(3 \alpha-7)$-provable but not $\alpha$-provable. For $\alpha=4$ this

Received by the editors October 27, 1988 and, in revised form, November 9, 1989.

1980 Mathematics Subject Classification (1985 Revision). Primary 03G15, 03 F20. 
yields the known result ([TG87, p. $\left.\left.93^{5}-93^{8}\right]\right)$ that there is a 3-sentence which is 5-provable but not 4-provable. For $\alpha=5$ it yields a 3-sentence which is 8provable but not 5-provable. However, by another construction we know there is a 6-provable 3-sentence which is not 5-provable. This construction also yields the result $\phi(5)=6$ mentioned above.

Theorem 1. Let $3<\alpha<\omega$. Then $\mathrm{SNr}_{3} \mathrm{CA}_{\alpha} \neq \mathrm{SNr}_{3} \mathrm{CA}_{3 \alpha-7}$. In fact, there is a simple finite relation algebra $\mathfrak{A}$ such that

(i) $B_{\alpha} \mathfrak{A}$ is an $\alpha$-dimensional cylindrical basis for $\mathfrak{A}$,

(ii) $\mathfrak{A}$ has no $(3 \alpha-7)$-dimensional relational basis,

(iii) $\mathfrak{A} \in \mathfrak{R a}^{*} C A_{\alpha}$,

(iv) $\mathfrak{C a}_{3} \mathfrak{A} \in \mathrm{Nr}_{3} \mathrm{CA}_{\alpha}$,

(v) $\mathfrak{A} \notin \mathbf{S} \mathfrak{R a}{ }^{*} \mathrm{CA}_{3 \alpha-7}$,

(vi) $\mathrm{CaB}_{3} \mathfrak{A} \notin \mathrm{SNr}_{3} \mathrm{CA}_{3 \alpha-7}$.

Proof. Let $\beta=3 \alpha-7$. Let $\mathfrak{B}=\langle B,+,-\rangle$ be a finite Boolean algebra which has the following atoms:

$d_{\kappa}$ and $e_{\kappa}$ for $\kappa<\beta$,

$a_{\kappa \lambda}^{\Gamma}$, where $\kappa, \lambda<\beta, \kappa \neq \lambda, \Gamma \subseteq \beta, \quad$ and either $\Gamma=\varnothing$ or $|\Gamma|=\alpha-2$.

Let $A t \mathfrak{B}$ be the set of atoms of $\mathfrak{B}$. We need some terminology for the indices used in the notation of the atoms. The first index of the atoms $d_{\kappa}, e_{\kappa}$, and $a_{\kappa \lambda}^{\Gamma}$ is $\kappa$, and the second index of $d_{\lambda}, e_{\lambda}$, and $a_{\kappa \lambda}^{\Gamma}$ is $\lambda$. (It may be perhaps clearer to use the notation $d_{\kappa \kappa}$ and $e_{\kappa \kappa}$ in place of $d_{\kappa}$ and $e_{\kappa}$.) Define an operation " on $A t \mathfrak{B}$ as follows.

$$
\check{d}_{\kappa}=d_{\kappa}, \check{e}_{\kappa}=e_{\kappa}, \quad \text { and } \quad\left(a_{\kappa \lambda}^{\Gamma}\right)^{\check{L}}=a_{\lambda \kappa}^{\Gamma} .
$$

Extend this operation to all the elements of $\mathfrak{B}$ :

$$
\check{x}=\sum\{\check{a}: x \geq a \in A t \mathfrak{B}\} .
$$

Let $C$ be the set consisting of all the following triples of atoms of $\mathfrak{B}$ :

$$
\begin{aligned}
& \left\langle d_{\kappa}, d_{\kappa}, d_{\kappa}\right\rangle,\left\langle e_{\kappa}, e_{\kappa}, e_{\kappa}\right\rangle,\left\langle d_{\kappa}, e_{\kappa}, e_{\kappa}\right\rangle,\left\langle e_{\kappa}, d_{\kappa}, e_{\kappa}\right\rangle,\left\langle e_{\kappa}, e_{\kappa}, d_{\kappa}\right\rangle, \\
& \left\langle d_{\kappa}, a_{\kappa \lambda}^{\Gamma}, a_{\kappa \lambda}^{\Gamma}\right\rangle,\left\langle a_{\kappa \lambda}^{\Gamma}, d_{\lambda}, a_{\kappa \lambda}^{\Gamma}\right\rangle,\left\langle a_{\kappa \lambda}^{\Gamma}, a_{\lambda \kappa}^{\Gamma}, d_{\kappa}\right\rangle, \\
& \left\langle e_{\kappa}, a_{\kappa \lambda}^{\Gamma}, a_{\kappa \lambda}^{\Delta}\right\rangle,\left\langle a_{\kappa \lambda}^{\Gamma}, e_{\lambda}, a_{\kappa \lambda}^{\Delta}\right\rangle,\left\langle a_{\kappa \lambda}^{\Gamma}, a_{\lambda \kappa}^{\Delta}, e_{\kappa}\right\rangle, \\
& \left\langle a_{\kappa \lambda}^{\Gamma}, a_{\lambda \mu}^{\Delta}, a_{\kappa \mu}^{\Sigma}\right\rangle, \text { where } \lambda \in \Sigma \text { or } \kappa \in \Delta \text { or } \mu \in \Gamma, \\
& \left\langle a_{0 \lambda}^{\varnothing}, a_{\lambda 1}^{\varnothing}, a_{01}^{\varnothing}\right\rangle,\left\langle a_{1 \lambda}^{\varnothing}, a_{\lambda 0}^{\varnothing}, a_{10}^{\varnothing}\right\rangle,\left\langle a_{01}^{\varnothing}, a_{1 \lambda}^{\varnothing}, a_{0 \lambda}^{\varnothing}\right\rangle,\left\langle a_{10}^{\varnothing}, a_{0 \lambda}^{\varnothing}, a_{1 \lambda}^{\varnothing}\right\rangle, \\
& \left.\qquad a_{\lambda 0}^{\varnothing}, a_{01}^{\varnothing}, a_{\lambda 1}^{\varnothing}\right\rangle,\left\langle a_{\lambda 1}^{\varnothing}, a_{10}^{\varnothing}, a_{\lambda 0}^{\varnothing}\right\rangle, \text { where } 1<\lambda<\beta .
\end{aligned}
$$

Then define a binary operation ; on $\mathfrak{B}$ by

$$
x ; y=\sum\{c: x \geq a \in A t \mathfrak{B}, y \geq b \in A t \mathfrak{B},\langle a, b, c\rangle \in C\} .
$$

Set $1^{\prime}=\sum_{\kappa<\beta} d_{\kappa}$, and let $\mathfrak{A}=\left\langle B,+,-, ;,,^{\prime}\right\rangle$. 
Next we will prove that $\mathfrak{A} \in W A$. From the way that ${ }^{-}$and ; are extended to all the elements of $\mathfrak{B}$, it follows that $\mathfrak{A}$ is isomorphic to the complex algebra

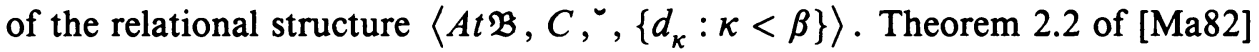
gives necessary and sufficient conditions for the complex algebra of such a structure to be an algebra in WA, so we need only verify those conditions. It is easy to see, from an inspection of the triples in $C$, that if $\langle x, y, z\rangle \in C$, then $\langle\breve{x}, z, y\rangle \in C$ and $\langle z, \breve{y}, x\rangle \in C$. Thus conditions (a) and (b) of Theorem 2.2 hold. Furthermore, if $\left\langle d_{k}, x, y\right\rangle \in C$ then $x=y$, and for any $x \in A t \mathfrak{B}$, there is some $\kappa<\beta$ such that $\left\langle d_{k}, x, x\right\rangle \in C$. Thus condition (c) of Theorem 2.2 holds, and we conclude that $\mathfrak{A} \in$ NA. To prove that condition (d) holds, and that therefore $\mathfrak{A} \in W A$, we need only assume $\left\langle d_{k}, x, x\right\rangle,\langle x, y, z\rangle \in C$ and show that $\left\langle d_{k}, z, z\right\rangle \in C$. Notice that all the triples in $C$ have the property that the first index of the first atom in a triple is the same as the first index of the last atom in that triple. Thus the first index of $d_{\kappa}$, namely $\kappa$, is the same as the first index of $x$, which is in turn the same as the first index of $z$. So the first index of $z$ is $\kappa$, and either $z=d_{k}$, or $z=e_{k}$, or $z=a_{\kappa \lambda}^{\Gamma}$ for some $\lambda, \Gamma$. In all three cases it happens that $\left\langle d_{\kappa}, z, z\right\rangle \in C$. Thus $\mathfrak{A} \in$ WA.

Next we show that $B_{\alpha} \mathfrak{A}$ is an $\alpha$-dimensional cylindrical basis for $\mathfrak{A}$. To do so we must verify conditions $\left(C_{0}\right),\left(C_{1}\right)$, and $\left(C_{2}\right)$ of Definition 4 in [Ma89]. First, condition $\left(C_{0}\right)$ follows from the fact that $\mathfrak{A} \in W A$, by Lemma 5 of [Ma89]. Some very simple computations serve to show that condition $\left(\mathrm{C}_{2}\right)$ holds when $M=B_{\alpha} \mathfrak{A}$. Thus what remains to show is $\left(\mathrm{C}_{1}\right)$. To do so, we assume $u, w \in B_{\alpha} \mathfrak{A}, \kappa, \lambda<\alpha, \kappa \neq \lambda$, and $u$ agrees with $w$ off of $\kappa, \lambda$. Then we must show that there is some $v$ in $B_{\alpha} \mathfrak{A}$ which agrees with $u$ off of $\kappa$, and agrees with $w$ off of $\lambda$. These required conditions determine all the values of $v$ except $v_{\kappa \lambda}$ and $v_{\lambda \kappa}$ as follows:

$$
v_{\mu \nu}= \begin{cases}u_{\mu \nu}=w_{\mu \nu} & \text { if } \kappa, \lambda \neq \mu, \nu<\alpha, \\ u_{\mu \nu} & \text { if } \kappa \neq \mu, \nu<\alpha, \\ w_{\mu \nu} & \text { if } \lambda \neq \mu, \nu<\alpha .\end{cases}
$$

Then $v_{\kappa \lambda}$ and $v_{\lambda \kappa}$ may be chosen as follows. In each case, the verification that $v \in B_{\alpha} \mathfrak{A}$ is routine.

Case 1. If $w_{\kappa \mu} \leq 1$, for some $\mu \in \alpha \sim\{\kappa, \lambda\}$, then $v_{\kappa \lambda}=u_{\mu \lambda}$ and $v_{\lambda \kappa}=u_{\lambda \mu}$. Case 2. If $u_{\mu \lambda} \leq 1$, for some $\mu \in \alpha \sim\{\kappa, \lambda\}$, then $v_{\kappa \lambda}=w_{\kappa \mu}$ and $v_{\lambda \kappa}=w_{\mu \kappa}$.

Case 3. Assume $w_{\kappa \mu}+u_{\mu \lambda} \leq 0^{\prime}$ whenever $\mu \in \alpha \sim\{\kappa, \lambda\}$. Let $\rho$ be the first index of $u_{\kappa \kappa}$. (Since $u_{\kappa \kappa} \leq 1, \rho, \rho$ is also the second index of $u_{\kappa \kappa}$.) For every $\mu \in \alpha \sim\{\kappa, \lambda\}$, we have $u_{\kappa \mu} \leq u_{\kappa \kappa} ; u_{\kappa \mu}$ since $u \in B_{\alpha} \mathfrak{A}$, hence $\left\langle u_{\kappa \kappa}, u_{\kappa \mu}, u_{\kappa \mu}\right\rangle \in C$. It follows that $\rho$ is the first index of $u_{\kappa \mu}$. Let $\sigma$ be the second (and first) index of $w_{\lambda \lambda}$. By similar reasoning, we get that $\sigma$ is the second index of $w_{\mu \lambda}$ for every $\mu \in \alpha \sim\{k, l\}$. If $\sigma=\rho$, we simply let $v_{\kappa \lambda}=v_{\lambda \kappa}=e_{\sigma}=e_{\rho}$. We may therefore assume $\sigma \neq \rho$. We will let $v_{\kappa \lambda}=a_{\rho \sigma}^{\Gamma}$ 
and $v_{\lambda \kappa}=a_{\sigma \rho}^{\Gamma}$ for some appropriately chosen $\Gamma$, to which we now turn.

Let $\mu \in \alpha \sim\{\kappa, \lambda\}$. Then $u_{\kappa \mu} \leq u_{\kappa \mu} ; u_{\mu \mu}$, so $\left\langle u_{\kappa \mu}, u_{\mu \mu}, u_{\kappa \mu}\right\rangle \in C$. Notice that all the triples in $C$ have the property that the second index of the second atom in a triple is the same as the second index of the last atom in that triple. Thus $u_{\mu \mu}$ and $u_{\kappa \mu}$ have the same second index. Similarly, $w_{\mu \mu}$ and $w_{\mu \lambda}$ have the same first index. But the first and second indices of $u_{\mu \mu}$ and $w_{\mu \mu}$ are all the same, since $u_{\mu \mu}=w_{\mu \mu} \leq 1$ '. Thus the second index of $u_{\kappa \mu}$ is the same as the first index of $w_{\mu \lambda}$. Let that common index be $\tau$. Then we have one of the following three possibilities:

$$
\begin{array}{lll}
u_{\kappa \mu}=a_{\rho \tau}^{\Delta} & \text { and } & w_{\mu \lambda}=a_{\tau \sigma}^{\Sigma}, \\
u_{\kappa \mu}=a_{\rho \tau}^{\Delta} & \text { and } & w_{\mu \lambda}=e_{\tau}=e_{\sigma}, \\
u_{\kappa \mu}=e_{\rho}=e_{\tau} & \text { and } & w_{\mu \lambda}=a_{\tau \sigma}^{\Sigma},
\end{array}
$$

A comparison of these possibilities with the definition of $C$ reveals that if $\tau \in \Gamma$, then $\left\langle u_{\kappa \mu}, w_{\mu \lambda}, a_{\sigma \rho}^{\Gamma}\right\rangle \in C$. Now the index $\tau$ depends on the choice of $\mu \in \alpha \sim\{\kappa, \lambda\}$, but there are only $\alpha-2$ such choices, so there are at most $\alpha-2$ such indices. Take $\Gamma$ to be any subset of $\alpha$ which contains all the indices which arise in this way. Set $v_{\kappa \lambda}=a_{\sigma \rho}^{\Gamma}$ and $v_{\lambda \kappa}=a_{\rho \sigma}^{\Gamma}$. It follows that $v_{\kappa \lambda}=u_{\kappa \mu} ; w_{\mu \lambda}$ for every $\mu \in \alpha \sim\{\kappa, \lambda\}$, and from this it is easy to show that $v \in B_{\alpha} \mathfrak{A}$. Thus (i) holds. Applying (i) with $\alpha=4$, we get that $B_{4} \mathfrak{A}$ is a 4-dimensional cylindrical basis, which implies that $\mathfrak{A}$ is a relation algebra.

So far the argument works for all $\alpha \geq 3$. We need $\alpha>3$ in the proof of (ii), since the conclusion of (ii) contradicts (i) when $\alpha=3$. We prove (ii) by contradiction. Suppose that $M \subseteq B_{\beta} \mathfrak{A}$ is a $\beta$-dimensional relational basis for $\mathfrak{A}$. By $\left(\mathrm{R}_{0}\right)$ of Definition 3, [Ma89], there is some $u \in M$ such that $u_{01}=a_{01}^{\varnothing}$. Since $a_{01}^{\varnothing} \leq \prod_{1<\lambda<\beta} a_{0 \lambda}^{\varnothing} ; a_{\lambda 1}^{\varnothing}$, we can repeatedly apply $\left(\mathrm{R}_{1}\right)$, obtaining some $v \in M$ such that

$$
v_{01}=a_{01}^{\varnothing}, \quad v_{0 \lambda}=a_{0 \lambda}^{\varnothing}, \quad v_{\lambda 1}=a_{\lambda 1}^{\varnothing}, \quad \text { for } 1<\lambda<\beta .
$$

Note that $v$ is designed so that the first and second indices of $v_{\kappa \lambda}$ are $\kappa$ and $\lambda$, respectively. By the definition of $C$, if $\kappa, \lambda \in\{2, \ldots, \beta-1\}$ and $\kappa \neq \lambda$ then there is some nonempty $\Gamma$ such that $v_{\kappa \lambda}=a_{\kappa \lambda}^{\Gamma}$ and $0,1 \in \Gamma$. For every 3-element subset $\{\kappa, \lambda, \mu\}$ of $\{2, \ldots, \beta-1\}$, we have $v_{\kappa \lambda} ; v_{\lambda \mu} \geq v_{\kappa \mu}$, hence $\{\kappa, \lambda, \mu\}$ contains some 2-element subset, say $\{\kappa, \lambda\}$, such that $\mu \in \Gamma$, where $v_{\kappa \lambda}=a_{\kappa \lambda}^{\Gamma}$. Whenever this happens, we say that $\{\kappa, \lambda\}$ accounts for $\{\kappa, \lambda, \mu\}$. Every 3-element subset of $\{2, \ldots, \beta-1\}$ must be accounted for by one of its 2-element subsets. Since $|\Gamma|=\alpha-2$ and $0,1 \in \Gamma$, every 2-element subset of $\{2, \ldots, \beta-1\}$ can account for only $\alpha-4$ of the 3 -element subsets of $\{2, \ldots, \beta-1\}$. But $(\alpha-4)\left(\begin{array}{c}\beta-2 \\ 2\end{array}\right)<\left(\begin{array}{c}\beta-2 \\ 3\end{array}\right)$, so we have a contradiction. Therefore $\mathfrak{A}$ has no $\beta$-dimensional relational basis, i.e., (ii) holds.

From the fact that $B_{\alpha} \mathfrak{A}$ is an $\alpha$-dimensional cylindrical basis for $\mathfrak{A}$, we conclude that $\mathfrak{C a}_{\alpha} \mathfrak{A} \in \mathrm{CA}_{\alpha}$ by Theorem 10(i), [Ma89]. Now $\mathfrak{A}$ is complete 
since it is finite, so we also have $\mathfrak{R a C a} B_{\alpha} \mathfrak{A} \cong \mathfrak{A}$ by Theorem10(iii), [Ma89]. Thus $\mathfrak{A} \cong \mathfrak{R a C a} B_{\alpha} \mathfrak{A} \in \mathfrak{R a}^{*} C A_{\alpha}$, so (iii) holds. It is straightforward to check that $\mathfrak{C a} B_{\gamma} \mathfrak{A} \cong \mathfrak{N r}_{\gamma} \mathfrak{C a} B_{\alpha} \mathfrak{A}$ whenever $3 \leq \gamma \leq \alpha$. In particular,

$$
\mathfrak{C a} B_{3} \mathfrak{A} \cong \mathfrak{N r}_{3} \mathfrak{C a} B_{\alpha} \mathfrak{A} \in \mathrm{Nr}_{3} \mathrm{CA}_{\alpha},
$$

so (iv) holds.

Now we prove (v) by contradiction. Suppose $\mathfrak{A} \in \mathrm{SRa}^{*} \mathrm{CA}_{\beta}$. Hence there is some $\mathfrak{C}$ such that

$$
\mathfrak{A} \subseteq \mathfrak{R a} \mathfrak{C}, \mathfrak{C} \in \mathrm{CA}_{\beta} .
$$

It turns out that (2) implies that $\mathfrak{A}$ has a $\beta$-dimensional relational basis. However, we will only show that (2) implies the existence of a $v \in B_{3} \mathfrak{A}$ satisfying (1), which leads to a contradiction, as shown above.

We have

so

$$
\begin{aligned}
0 & \neq a_{01}^{\varnothing} \cdot \prod_{1<\lambda<\beta} a_{0 \lambda}^{\varnothing} ; a_{\lambda 1}^{\varnothing} \\
& =a_{01}^{\varnothing} \cdot \prod_{1<\lambda<\beta} c_{\lambda}\left(s_{\lambda}^{1} a_{0 \lambda}^{\varnothing} \cdot s_{\lambda}^{0} a_{\lambda 1}^{\varnothing}\right) \\
& =c_{(\beta \sim 2)}\left(a_{01}^{\varnothing} \cdot \prod_{1<\lambda<\beta}\left(s_{\lambda}^{1} a_{0 \lambda}^{\varnothing} \cdot s_{\lambda}^{0} a_{\lambda 1}^{\varnothing}\right)\right),
\end{aligned}
$$

$$
0 \neq a_{01}^{\varnothing} \cdot \prod_{1<\lambda<\beta}\left(s_{\lambda}^{1} a_{0 \lambda}^{\varnothing} \cdot s_{\lambda}^{0} a_{\lambda 1}^{\varnothing}\right) .
$$

Define $\sigma_{\kappa \lambda}(x)$ for all $\kappa, \lambda<\beta$ and all $x \in N r_{2} \mathfrak{B}$ as follows:

$$
\sigma_{\kappa \lambda}(x)= \begin{cases}s_{\kappa}^{0} s_{\lambda}^{1} x & \text { if } \lambda \neq 0, \\ s_{0}^{1} s_{\kappa}^{0} x & \text { if } \lambda=0, \kappa \neq 1, \\ s_{0}^{2} s_{1}^{0} s_{2}^{1} x & \text { if } \lambda=0, \kappa=1 .\end{cases}
$$

Notice that $\sigma_{\kappa \lambda}(1)=1$ and $\sigma_{\kappa \lambda}$ is completely additive. Since $A t \mathfrak{A}$ is finite, we have

$$
1=\sum^{(\mathfrak{B})} A t \mathfrak{A}=\prod_{\kappa, \lambda<\beta} \sigma_{\kappa \lambda}\left(\sum A t \mathfrak{A}\right)=\sum_{v \in \in^{\beta \times \beta} A t \mathfrak{A}}\left(\prod_{\kappa, \lambda<\beta} \sigma_{\kappa \lambda}\left(v_{\kappa \lambda}\right)\right),
$$

so, by (3),

$$
\begin{aligned}
0 & \neq a_{01}^{\varnothing} \cdot \prod_{1<\lambda<\beta}\left(s_{\lambda}^{1} a_{0 \lambda}^{\varnothing} \cdot s_{\lambda}^{0} a_{\lambda 1}^{\varnothing}\right) \cdot 1 \\
& =a_{01}^{\varnothing} \cdot \prod_{1<\lambda<\beta}\left(s_{\lambda}^{1} a_{0 \lambda}^{\varnothing} \cdot s_{\lambda}^{0} a_{\lambda 1}^{\varnothing}\right) \cdot\left(\sum_{v \epsilon^{\beta \times \beta} A t \mathfrak{A}}\left(\prod_{\kappa, \lambda<\beta} \sigma_{\kappa \lambda}\left(v_{\kappa \lambda}\right)\right)\right) \\
& =\sum_{v \in^{\beta \times \beta} A t \mathfrak{A}}\left(a_{01}^{\varnothing} \cdot \prod_{1<\lambda<\beta}\left(s_{\lambda}^{1} a_{0 \lambda}^{\varnothing} \cdot s_{\lambda}^{0} a_{\lambda 1}^{\varnothing}\right) \cdot \prod_{\kappa, \lambda<\beta} \sigma_{\kappa \lambda}\left(v_{\kappa \lambda}\right)\right) .
\end{aligned}
$$


It follows that there is some $v \in{ }^{\beta \times \beta}$ At $\mathfrak{A}$ such that

$$
0 \neq a_{01}^{\varnothing} \cdot \prod_{1<\lambda<\beta}\left(s_{\lambda}^{1} a_{0 \lambda}^{\varnothing} \cdot s_{\lambda}^{0} a_{\lambda 1}^{\varnothing}\right) \cdot \prod_{\kappa, \lambda<\beta} \sigma_{\kappa \lambda}\left(v_{\kappa \lambda}\right) .
$$

By routine cylindric-algebraic computations it is now possible to show from (4) that $v \in B_{\beta} \mathfrak{A}$ and (1) holds. The proof is given in [Ma78, pp. $170^{8}-172^{5}$ ], for a slightly different definition of $\sigma_{\kappa \lambda}$. The existence of such a $v$ is contradictory, so (v) holds.

Finally, we prove (vi) by contradiction. Suppose $\mathfrak{C a} B_{3} \mathfrak{A} \in \mathrm{SNr}_{3} \mathrm{CA}_{\beta}$. Then $\mathfrak{R a C a} B_{3} \mathfrak{A} \in \mathfrak{R a}^{*} \mathrm{SNr}_{3} \mathrm{CA}_{\beta}$, but $\mathfrak{R a C a} B_{3} \mathfrak{A} \cong \mathfrak{A}$ by Theorem 10(iii), [Ma89], and $\mathfrak{R a}^{*} \mathrm{SNr}_{3} \mathrm{CA}_{\beta}=\mathrm{SRa}^{*} \mathrm{Nr}_{3} \mathrm{CA}_{\beta}=\mathbf{S R a}^{*} \mathrm{CA} A_{\beta}$ by [HMT85, 5.3.13], so $\mathfrak{A} \in$ $S \Re a^{*} C A_{\beta}$ contradicting (v). Thus (vi) holds.

For every natural number $n$ we let $\mathscr{M}^{(n)}$ be the first-order formalism defined in [TG87], §7.1. In more detail, $\mathscr{M}^{(n)}$ is a first-order language with equality symbol $\mathbf{1}$ and $n+1$ binary relation symbols $\mathbf{F}_{0}, \ldots, \mathbf{F}_{n} . \mathbf{\Upsilon}=\left\{\mathbf{v}_{\kappa}: \kappa<\omega\right\}$, $\Phi^{(n)}$, and $\Sigma^{(n)}$ are the sets of variables, formulas, and sentences of $\mathscr{M}^{(n)}$, respectively. [X] is the universal closure of $X \in \Phi^{(n)}$. The logical axiom schemata of $\mathscr{M}^{(n)}$ are (AI)-(AIX) ([TG87, p. 8]). $\Theta \eta^{(n)} \Psi$ is the $\mathscr{M}^{(n)}$-theory generated by $\Psi . \mathscr{M}^{(n)+}$ is the extended formalism obtained from $\mathscr{M}^{(n)}$ by adding certain operators on predicates and an additional equality symbol $=$. $\Pi^{(n)}, \boldsymbol{\Phi}^{(n)+}$, and $\Sigma^{(n)+}$ are the sets of predicates, formulas, and sentences of $\mathscr{M}^{(n)+}$, respectively. The logical axiom schemata of $\mathscr{M}^{(n)+}$ are (AI)-(AIX) and also (DI)-(DV) ([TG87], p. 25). For $3 \leq \alpha<\omega$, the formalisms $\mathscr{M}_{\alpha}^{(n)}$ and $\mathscr{M}_{\alpha}^{(n)+}$ are the fragments of $\mathscr{M}^{(n)}$ and $\mathscr{M}^{(n)+}$ obtained by restricting to only the first $\alpha$ variables $\mathbf{v}_{0}, \ldots, \mathbf{v}_{\alpha-1}$. Their definitions require some additional axiom schemata, namely $\left(\mathrm{AIX}^{\prime}\right),\left(\mathrm{AIX}^{\prime \prime}\right),(\mathrm{AX})$, and $\left(\mathrm{AX}^{\prime}\right)$ ([TG87, pp. 68, 70]). The sets of formulas and sentences of these fragments are $\boldsymbol{\Phi}_{\alpha}^{(n)}, \boldsymbol{\Phi}_{\alpha}^{(n)+}$, $\Sigma_{\alpha}^{(n)}$, and $\boldsymbol{\Sigma}_{\alpha}^{(n)+}$. The theories in $\mathscr{M}_{\alpha}^{(n)}$ and $\mathscr{M}_{\alpha}^{(n)+}$ which are generated by $\Psi$ are $\Theta \eta_{\alpha}^{(n)} \Psi$ and $\Theta \eta_{\alpha}^{(n)+} \Psi$. As noted in [TG87], all the results for $\mathscr{L}$ and $\mathscr{L}^{+}$ carry over to $\mathscr{M}^{(n)}$ and $\mathscr{M}^{(n)+}$ with only minor notational modifications.

Theorem 2. If $4 \leq \alpha<\omega$ then $\varnothing \neq \Sigma_{3}^{(n)} \cap\left(\Theta \eta_{3 \alpha-7}^{(n)} \varnothing \sim \theta \eta_{\alpha}^{(n)} \varnothing\right)$ and $\varnothing \neq$ $\Sigma_{3}^{(n)+} \cap\left(\Theta \eta_{3 \alpha-7}^{(n)+} \varnothing \sim \Theta \eta_{\alpha}^{(n)+} \varnothing\right)$.

Proof. Set $\beta=3 \alpha-7$. By Theorem 1 there is a finite relation algebra $\mathfrak{A}$ with an $\alpha$-dimensional cylindrical basis but no $\beta$-dimensional relational basis. Let $n+1=|A t \mathfrak{A}|$. Let $f=\left\langle f_{i}: i \leq n\right\rangle$ be an enumeration of the atoms of $\mathfrak{A}$. Let $\mathfrak{M}=\left\langle\mathfrak{A}, f, B_{\alpha} \mathfrak{A}\right\rangle$. Then $\mathfrak{M}$ is an $\alpha$-dimensional algebraic realization of both $\mathscr{M}^{(n)}$ and $\mathscr{M}^{(n)+}$, in the sense of Definition 16, [Ma89], since $B_{\alpha} \mathfrak{A}$ is an $\alpha$-dimensional cylindrical basis and clearly $\left(\mathrm{C}_{3}\right)$ of Definition 16 holds. (Note that Definitions 16 and 17 should be slightly modified to account for the fact that $\mathscr{M}^{(n)}$ and $\mathscr{M}^{(n)+}$ have many atomic predicates instead of just one. 
All the results of [Ma89] continue to hold without any further changes.) By Theorem 22(iii), [Ma89], every $X \in \Theta \eta_{\alpha}^{(n)+} \varnothing$ is algebraically true of $\mathfrak{M}$.

Consider the statement

$$
\text { every } X \in \Theta \eta_{\beta}^{(n)+} \varnothing \quad \text { is algebraically true of } \mathfrak{M} \text {. }
$$

It can be shown (by imitating the proof of Lemma 4.3.22 of [HMT85]) that (5) implies (2) for some $\mathfrak{C}$. Since (2) implies the existence of a $v \in B_{\beta} \mathfrak{A}$ such that (1), we have a contradiction, and the proof is complete. However, we can also obtain a sentence $X \in \Sigma_{3}^{(n)}$ which is algebraically false in $\mathfrak{M}$ (hence $\left.X \notin \Theta \eta_{\alpha}^{(n)+} \varnothing\right)$, such that one can show directly (by constructing a $\beta$-proof) that $X \in \Theta \eta_{\beta}^{(n)+} \varnothing$. Let $Y$ be the conjunction of the following sentences:

$$
\begin{aligned}
& \forall \mathbf{v}_{0} \forall \mathbf{v}_{1}\left(\mathbf{F}_{i} \mathbf{v}_{0} \mathbf{v}_{1} \rightarrow \neg \mathbf{F}_{j} \mathbf{v}_{0} \mathbf{v}_{1}\right) \text { for } i, j \leq n, i \neq j, \\
& \forall \mathbf{v}_{0} \forall \mathbf{v}_{1}\left(\bigvee_{i \leq n} \mathbf{F}_{i} \mathbf{v}_{0} \mathbf{v}_{1}\right), \\
& \forall \mathbf{v}_{0} \forall \mathbf{v}_{1}\left(\mathbf{F}_{i} \mathbf{v}_{0} \mathbf{v}_{1} \leftrightarrow \mathbf{F}_{j} \mathbf{v}_{1} \mathbf{v}_{0}\right) \quad \text { whenever } f_{i}^{\sim}=f_{j}, i, j \leq n \text {, } \\
& \forall \mathbf{v}_{0} \forall \mathbf{v}_{1}\left(\mathbf{v}_{0} \mathbf{i} \mathbf{v}_{1} \leftrightarrow \bigvee_{f_{i} \leq 1} \mathbf{F}_{i} \mathbf{v}_{0} \mathbf{v}_{1}\right), \\
& \forall \mathbf{v}_{0} \forall \mathbf{v}_{1}\left(\mathbf{F}_{i} \mathbf{v}_{0} \mathbf{v}_{1} \rightarrow \exists \mathbf{v}_{2}\left(\mathbf{F}_{j} \mathbf{v}_{0} \mathbf{v}_{2} \wedge \mathbf{F}_{k} \mathbf{v}_{2} \mathbf{v}_{1}\right)\right) \\
& \text { whenever } i, j, k \leq n \text { and } f_{i} \leq f_{j} ; f_{k} \text {, } \\
& \forall \mathbf{v}_{0} \forall \mathbf{v}_{1}\left(\mathbf{F}_{i} \mathbf{v}_{0} \mathbf{v}_{1} \rightarrow \neg \exists \mathbf{v}_{2}\left(\mathbf{F}_{j} \mathbf{v}_{0} \mathbf{v}_{2} \wedge \mathbf{F}_{k} \mathbf{v}_{2} \mathbf{v}_{1}\right)\right) \\
& \text { whenever } i, j, k \leq n \text { and } f_{i} \cdot f_{j} ; f_{k}=0 \text {. }
\end{aligned}
$$

$Y$ encodes the structure of $\mathfrak{A}$ in such a way that if $Y$ has a model $\mathfrak{U}=$ $\left\langle U, F_{i}\right\rangle_{i \leq n}$ then there is an embedding of $\mathfrak{A}$ into the relation algebra of all binary relations on $U$ mapping each atom $f_{i}$ to the interpretation $F_{i}$ of $\mathbf{F}_{i}$. Let $X=\neg Y$. By a tedious decipherment of Definition 17, [Ma89], one can show that $Y$ is algebraically true of $\mathfrak{M}$, and therefore $X$ is not algebraically true of $\mathfrak{M}$. On the other hand, $\mathfrak{A}$ is not representable (since it is finite and has no $\beta$-dimensional relational basis), so $Y$ is inconsistent and $X$ is logically valid. The fact that $\mathfrak{A}$ has no $\beta$-dimensional relational basis implies that there is a proof of $X$ in $\mathscr{K}_{\beta}^{(n)}$. To see why this is so, let us suppose that $\mathfrak{A}$ actually coincides with the algebra constructed in the proof of Theorem 1. Then a proof of $X$ in $\mathscr{M}_{\beta}^{(n)}$ can be modeled on part of the proof of Theorem 1. To get started, first note that $Y$ has the following consequence:

$$
\begin{array}{r}
\exists \mathbf{v}_{0} \exists \mathbf{v}_{1}\left(\mathbf { F } _ { i } \mathbf { v } _ { 0 } \mathbf { v } _ { 1 } \wedge \bigwedge \left\{\exists \mathbf{v}_{2}\left(\mathbf{F}_{j} \mathbf{v}_{0} \mathbf{v}_{2} \wedge \mathbf{F}_{k} \mathbf{v}_{2} \mathbf{v}_{1}\right):\right.\right. \\
\left.\left.1<\lambda<\beta \& a_{0 \lambda}^{\varnothing}=f_{j} \& a_{\lambda 1}^{\varnothing}=f_{k}\right\}\right) .
\end{array}
$$


This sentence can be put into prenex normal form with quantifier prefix $\exists \mathbf{v}_{0} \exists \mathbf{v}_{1}$ $\ldots \exists \mathbf{v}_{\beta-1}$ and a matrix corresponding to (1). The conjuncts of $Y$ can then be used to show that the matrix is inconsistent. The combinatorial reason for this inconsistency is expressed by the part of the proof of Theorem 1 concerning the 2-element and 3-element subsets of $\{2, \ldots, \beta-1\}$. Of course, the counting argument used there cannot be translated directly into a proof in $\mathscr{M}_{\beta}^{(n)}$. The cases must be listed and individually ruled out. Thus the proof will be straightforward but of enormous length.

\section{REFERENCES}

[HMT71] Leon Henkin, J. Donald Monk, and Alfred Tarski, Cylindric algebras, Part I, NorthHolland, Amsterdam, 1971.

[HMT85] _ C Cylindric algebras, part II, North-Holland, Amsterdam, 1985.

[J69] J. Johnson, Nonfinitizability of classes of representable polyadic algebras, J. Symbolic Logic 34 (1969), 344-352.

[Ma78] Roger D. Maddux, Topics in relation algebras, Doctoral dissertation, University of California, Berkeley, 1978, pp. iii+241.

[Ma82] _ Some varieties containing relation algebras, Trans. Amer. Math. Soc. 272 (1982), 501-526.

[Ma85] _ Finite integral relation algebras, Universal Algebra and Lattice Theory (Proc. Southeastern Conf. in Universal Algebra and Lattice Theory, July 11-14, 1984), Lecture Notes in Math., vol. 1149, Springer-Verlag, Berlin, 1985, pp. 175-197.

[Ma89] __ Nonfinite axiomatizability results for cylindric and relation algebras, J. Symbolic Logic (3) 54 (September 1989), 951-974.

[Mo69] J. Donald Monk, Nonfinitizability of classes of representable cylindric algebras, J. Symbolic Logic 34 (1969), 331-343.

[Mo71] _ Provability with finitely many variables, Proc. Amer. Math. Soc. 27 (1971), 353-358.

[TG87] Alfred Tarski and Steven Givant, A formalization of set theory without variables, Amer. Math. Soc. Colloq. Publ. vol. 41, 1987.

Department of Mathematics, Iowa State University, Ames, Iowa 50011 